Article

\title{
Tensile Tests for the Improvement of Adhesion between Rubber and Steel Layers in Elastomeric Isolators
}

\author{
Margherita Pauletta *, Federico Pinzano, Giada Frappa and Gaetano Russo \\ Polytechnic Department of Engineering and Architecture, University of Udine, 33100 Udine, Italy; \\ pinzano.federico@spes.uniud.it (F.P.); giada.frappa@uniud.it (G.F.); gaetano.russo@uniud.it (G.R.) \\ * Correspondence: margherita.pauletta@uniud.it
}

Received: 5 October 2020; Accepted: 12 November 2020; Published: 13 November 2020

check for updates

\begin{abstract}
Steel reinforced elastomeric isolators are currently the most used bearings for seismic isolation purposes. The steel reinforcements are cut to the desired shape, sandblasted, cleaned with acid, and coated with bonding compound during the manufacturing process. Then the elastomer and steel layers are stacked in a mold and subjected to vulcanization so that they are glued together and constitute a single body. Good adhesion between the layers is very important for the correct functioning of the device. Adhesion conditions become critical when the isolators are subjected to tensile stresses, which arise under direct tensile actions or large shear strains. To analyze the influence of changes in the manufacturing process on the isolator adhesive behavior, the authors performed tensile tests on square-shaped small-scale specimens rather than expensive shear tests on full-scale isolators. Hence, the adhesion behavior between elastomer and steel layers was investigated through the tensile tests discussed herein. Among the influencing parameters that were considered, it was found that an increase in vulcanization time does not improve the adhesion, but it may actually worsen the capacity of the isolator in terms of strength. Moreover, it was found that using elastomer without an oily component improves the adhesion between the layers and increases the isolator's dissipative capacity.
\end{abstract}

Keywords: elastomeric isolators; adhesion; vulcanization time; corundum sandblasting; adhesive rubber; natural rubber; synthetic rubber; dissipative compound; non-dissipative compound

\section{Introduction}

Steel reinforced elastomeric isolators (SREIs) are currently the most used bearings for seismic isolation purposes. They are made with steel reinforcing layers (2-3 $\mathrm{mm}$ thick) alternating with layers of elastomer (5-20 mm thick), which are bonded together by means of a bonding compound and a vulcanization process. These isolators are delimited at the top and at the bottom by two $25-30 \mathrm{~mm}$ thick steel endplates, which are anchored to the isolator steel end layers and anchor the isolator to the sub- and super-structure.

In the process of producing the SREI core, i.e., the central part of the isolator without the thick steel end plates, the steel reinforcements are cut into the desired shape, sandblasted, cleaned with acid, and coated with bonding compound. The sandblasting roughens the steel surface to facilitate bonding with the elastomer. The bonding compound is made of two chemical substances: the primer and the mastic. The primer, which is applied directly to the steel, is meant to improve adhesion between the steel and mastic. The mastic is applied over the primer and glues the elastomer layer to the steel plates. The steel and the elastomer layers are then stacked in a mold and subjected to a pre-determined compression at a temperature and pressure that causes them to be glued together by means of vulcanization, so that they are held together and constitute a single body. 
Good adhesion is very important in all rubber composite devices for the correct functioning of materials bonded to the elastomer [1-9]. Moreover, it is important, because the theoretical calculus of rubber composite devices is based on the hypothesis of perfect bonding between the elastomer and the other materials $[1,2,10]$.

The adhesion conditions of the steel and elastomer layers, obtained in SREIs as described above, become critical under two particular conditions:

- the isolator is subjected to direct tensile stresses, i.e., a tensile action is present

- the isolator is subjected to large shear strains, which induce tensile stresses in the isolator extremities bounded by the white dotted line shown in Figure 1.

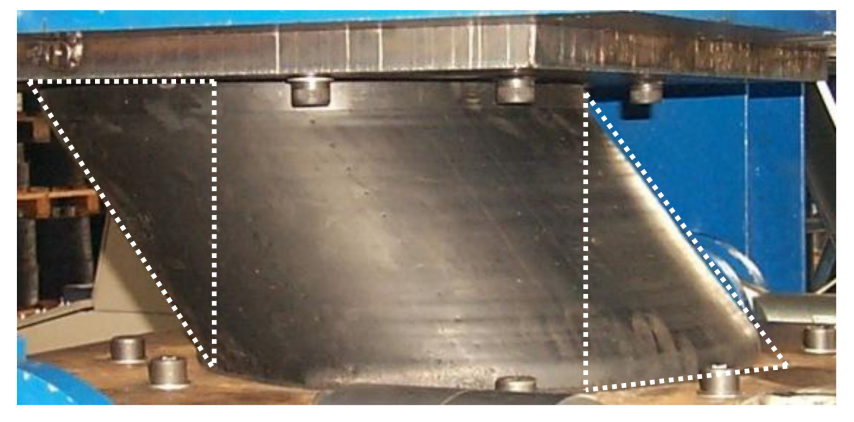

Figure 1. Cylindrical elastomeric isolator under shear strains.

The first condition may occur in tall buildings [11], which undergo large overturning moments that induce tensile stresses on the isolators and uplift of the building.

However, this is not the only situation in which isolators undergo tensile stress.

Keda and Nagahashi [12] investigated the earthquake response characteristics of the isolators in a base-isolated high-rise building with a set-back and found that axial stresses in isolators at the corners below the low-rise part are higher than those in isolators under the high-rise part, with some strong motions inducing maximum tensile stresses exceeding $1 \mathrm{MPa}$.

Mitoulis [13] performed an extended parametric study that revealed that bearing uplift due to tensile action may also occur in isolated bridges, and this effect is more critical for the bearings on shorter piers. This finding was also confirmed by Tubaldi et al. [14].

The standard UNI EN 15129 [15] takes into account the possibility that the isolators are able to undergo tensile stress; however, to avoid the cavitation of the rubber the value of this load is limited to $2 \mathrm{G}$, where $\mathrm{G}$ is the isolator shear modulus measured at a $100 \%$ strain. The strain is calculated as the ratio between the maximum applied displacement and the isolator rubber total thickness. Since the G values provided by the isolator manufacturers generally range from $0.4 \mathrm{MPa}$ to $1.4 \mathrm{MPa}$, the above limit ranges from $0.8 \mathrm{MPa}$ to $2.8 \mathrm{MPa}$.

The second condition is where tensile stress arises in the isolator due to large shear strains, even if the isolator is subjected to compression.

Kalfas et al. [16] observed that when a rotational restraint is applied on the anchor plates of the bearings, which is the case in vertically heavily-loaded buildings, under shear strains in the range of $125-375 \%$, compressive stresses develop in the regions closer to the center of the bearing while tensile stresses are observed at the edges of the rubber layers bounded by the white dotted line shown in Figure 1.

To take this phenomenon into account, the standards impose severe shear tests on the isolators during the device qualification stage.

In standard UNI EN 15129 [15], the tests provided to assess the performance requirements for elastomeric isolators involve the isolators being subjected to increasing maximum shear strains up to a value of 2.5. Under this very demanding condition, the isolator extremities (bounded by the white dotted line in Figure 1) are subjected to tensile stresses. If the bonding between the steel and the 
rubber layers is not well realized, these stresses may induce a phenomenon called delamination, which involves the detachment of the steel layers from the elastomeric ones.

In the first stage of production, a new isolator is subjected to many tests at the maximum shear strain before it attains the required isolator performance and is qualified to be placed on the market.

These shear tests are quite expensive because they require the use of a specific machine, and moreover, a number of isolators, which are individually expensive. Therefore, it would be helpful for manufacturers if they could perform less expensive tests on a number of small-scale simple specimens using a less specific machine, for example, a tension-compression test machine used for testing materials.

The authors were prompted to consider this issue when an isolator manufacturer requested an investigation of the adhesion between elastomer and steel layers of isolators while varying some of the manufacturing processes. Various manufacturing processes could be changed one at a time to study their individual influence on the isolator performance. Hence, a large number of isolators would need to be produced and subjected to testing, which would involve considerable expense. To reduce this expense and conduct preliminary investigations, the authors proposed that the tensile tests be performed on square-shaped small-scale specimens.

The experimental procedures used to study the behavior of the bond between the steel and elastomeric layers of the isolator are described herein, and the influence of the changes in the manufacturing process on the isolator performance are discussed.

\section{Sample Characteristics}

The isolator prototypes were built by ILPEA Industries of Pordenone, Italy, and experimentally tested at the Laboratory for Testing Materials and Structures of the University of Udine, Italy. The samples were square-shaped and small, because this configuration results in the lowest cost for the manufacturer. The thickness of the elastomer and steel layers were realized in the same order of magnitude of those used for medium-size real isolators. The geometrical characteristics of the samples are shown in Figure 2.

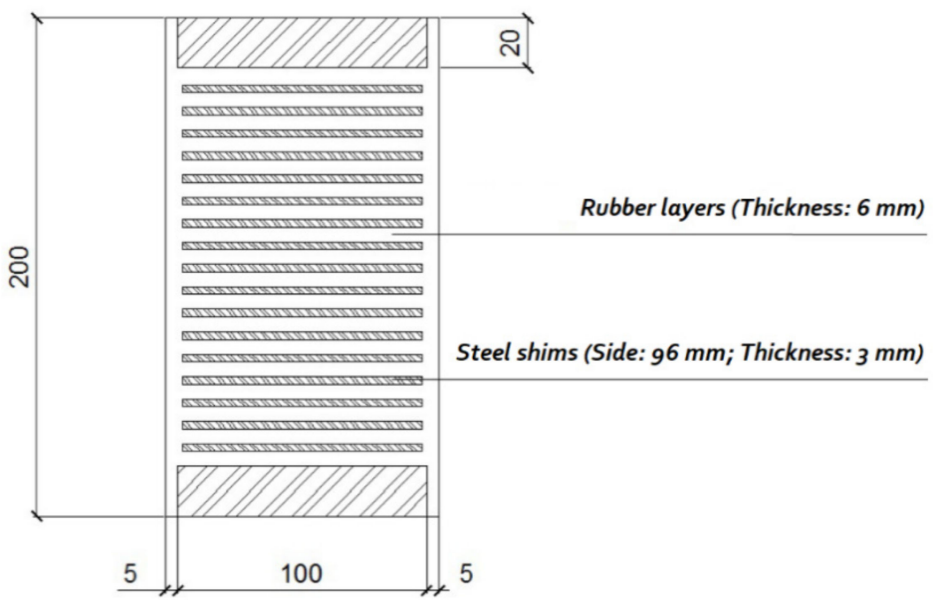

Figure 2. Geometrical characteristics of samples used for the tensile tests.

The samples were produced using a square-based mold, in which a $20 \mathrm{~mm}$ thick steel plate was placed at the bottom and an elastomer covering of $5 \mathrm{~mm}$ (if provided) was placed on the vertical lateral surfaces. Subsequently, $6 \mathrm{~mm}$ thick elastomer layers alternating with the $3 \mathrm{~mm}$ thick reinforcement elements, were inserted one on top of the other.

The steel reinforcements were sandblasted and pre-treated with primer and mastic. The sandblasting roughens the steel surface to facilitate bonding. The primer, applied directly to the steel, is a chemical substance that is meant to improve the adhesion between steel and mastic. The 
mastic, a liquid glue. is applied over the primer to paste the elastomer layer to the steel reinforcement. After the whole mold is filled with these layers, the next operation is the vulcanization. During vulcanization the layers are subjected, for certain period of time, to a pre-determined compression at a temperature that causes them to glue to each other, so that the layers are held together and substantially constitute a single body. The vulcanization time varies, depending on the dimensions of the isolator.

The characteristics of all 24 tested samples are reported in Table 1.

Elastomer layers with three different shear modulus, $\mathrm{G}=0.4 \mathrm{MPa}, \mathrm{G}=0.8 \mathrm{MPa}$ and $\mathrm{G}=1.4 \mathrm{MPa}$, were used. Different types of compound can be present in a group of samples with the same shear modulus. The compounds that were considered and their characteristics are:

- 35,713-hardness 70, shore A, styrene butadiene rubber (SBR)-based, which is a synthetic rubber, dissipative compound

- 35,718-hardness 60, shore A, natural rubber (NR)-based, dissipative compound

- 34,821-hardness 40, shore A, NR-based, dissipative compound

- 312-hardness 70, shore A, SBR-based, non-dissipative compound

- 27,303-hardness 60, shore A, SBR-based, dissipative compound.

All of these compounds are used by ILPEA to produce seismic isolators, apart from compound 312 , which is used to produce road joints. This compound has good abrasion resistance and good elongation capacity, but it is not dissipative.

To investigate how the adhesion between elastomer and steel layers can vary, the following manufacturing processes were taken into consideration:

- The basic vulcanization time of the tested samples, considering their dimensions is $135 \mathrm{~min}$ according to ILPEA manufacturing standards. In the experimental campaign, this time was varied to investigate if the variation produced effects on the layers' adhesion. Longer vulcanization time involves longer pressure application and heating, which are the processes that result in the reorganization of the rubber compound crosslinks to obtain the final elastomer and the gluing of the elastomer to the steel layers. Time periods of 150 and $180 \mathrm{~min}$ are taken into consideration.

- Corundum was used for sandblasting instead of standard grit to produce some of the samples used in the study. Corundum is an aluminum oxide and it is used in the manufacture of abrasives because of its great hardness, which is close to that of diamond. It can also be produced synthetically. This material may be able to increase the roughness of steel reinforcements, and consequently, the adhesion between steel and elastomer layers.

- Isolators usually have an elastomer layer covering the lateral external surface. This layer is obtained by using steel reinforcements that have a diameter or side length smaller than the interior dimension of the mold. During the vulcanization, the elastomer of the horizontal layers occupies the spaces that remain empty near the surface of the mold, and thus creates the external covering. This covering protects the isolator core from external agents. For most of the considered samples, the thickness of the external covering was equal to $5 \mathrm{~mm}$, but some samples without external covering layer were also tested for comparison.

- In the isolator manufacturing process, the elastomer layers are sometimes left for a long time in the warehouse before being used to build isolators. During this storage period, an oily component of the elastomer tends to rise on the outer surface of the layer. Therefore, this oily component could partially compromise the adhesion of the elastomer to the mastic. For this reason, a number of samples were produced using elastomer without this component.

- Additionally, several samples were produced with elastomer layers that had been refreshed before being put into the mold for vulcanization, in order to eliminate the problem of oily component on the surfaces.

- In some samples, a "bridge layer", which aims at increasing the elastomer-steel adhesion, was put between the mastic and the elastomer layer. 
- Moreover, considering samples with the same shear modulus of $0.8 \mathrm{MPa}$, three different elastomeric compounds: $35,718,312$ and 27,303 were used to investigate the influence of compound type on the adhesion behavior. The difference between compound 35,718 and 27,303 is in the type of rubber used to produce the elastomer, that is, NR for the former and SBR for the latter, while compound 312 is not dissipative, unlike compound 27,303.

In Table 1, samples indicated with a star are the "control samples" that were used for comparison with the results of the tests on the samples where the manufacturing processes was changed.

Sample 0 was used to calibrate the test instrumentation.

The samples labeled with the same number followed by letter $a$ or $b$ are identical to each other.

Table 1. Characteristics of the samples subjected to uniaxial tensile tests.

\begin{tabular}{|c|c|c|c|c|}
\hline Sample & Elastomer & $\mathrm{G}[\mathrm{MPa}]$ & $\mathrm{T}[\mathrm{min}]$ & Features \\
\hline $1 *$ & 35,713 & 1.4 & 135 & - \\
\hline 3 & 35,713 & 1.4 & 180 & - \\
\hline $4^{*}$ & 35,718 & 0.8 & 135 & - \\
\hline 5 & 35,718 & 0.8 & 150 & - \\
\hline 8 & 34,821 & 0.4 & 150 & - \\
\hline 9 & 34,821 & 0.4 & 180 & - \\
\hline 10 & 35,713 & 1.4 & 135 & Corundum sandblasting of steel. \\
\hline 11 & 312 & 0.8 & 135 & - \\
\hline $12 *$ & 27,303 & 0.8 & 135 & - \\
\hline $15 b$ & 35,713 & 1.4 & 135 & Without external covering layer, elastomer without oily component. \\
\hline $16 \mathrm{a}$ & 35,713 & 1.4 & 135 & Without external covering layer, refreshing of elastomer layers. \\
\hline $16 \mathrm{~b}$ & 35,713 & 1.4 & 135 & Without external covering layer, refreshing of elastomer layers. \\
\hline $17 \mathrm{a}$ & 35,713 & 1.4 & 135 & Without external covering layer, presence of bridge layer. \\
\hline $17 \mathrm{~b}$ & 35,713 & 1.4 & 135 & Without external covering layer, presence of bridge layer. \\
\hline $18 \mathrm{a}$ & 35,713 & 1.4 & 135 & $\begin{array}{c}\text { Without external covering layer, refreshing of elastomer layers, } \\
\text { presence of bridge layer. }\end{array}$ \\
\hline $18 b$ & 35,713 & 1.4 & 135 & $\begin{array}{c}\text { Without external covering layer, refreshing of elastomer layers, } \\
\text { presence of bridge layer. }\end{array}$ \\
\hline
\end{tabular}

\section{Experimental Investigation}

The experimental campaign investigated how the adhesion between elastomer and steel layers varies when the above-described manufacturing processes are varied. The aim of the investigation was to select those solutions where the sample failure occurred due to elastomer rupture and not for delamination of the elastomer layers from steel layers. This ensured that the sample achieved the highest possible strength.

Moreover, according to UNI EN 15129 [15], it is important that all specimens have a tensile strength greater than $2 \mathrm{G}$.

\subsection{Test Setup and Instrumentation}

A MTS machine (model 810) with a displacement capacity of $150 \mathrm{~mm}$ and a load capacity of $500 \mathrm{kN}$ (Figure 3) was used for the tensile tests. The specimens end plates were welded to two steel plates, which were disposed vertically and used to anchor the specimen to the jaws of the machine, as shown in Figure 3.

The tensile tests were carried out under displacement control, with an increasing quasi-static displacement applied until the sample failed. 


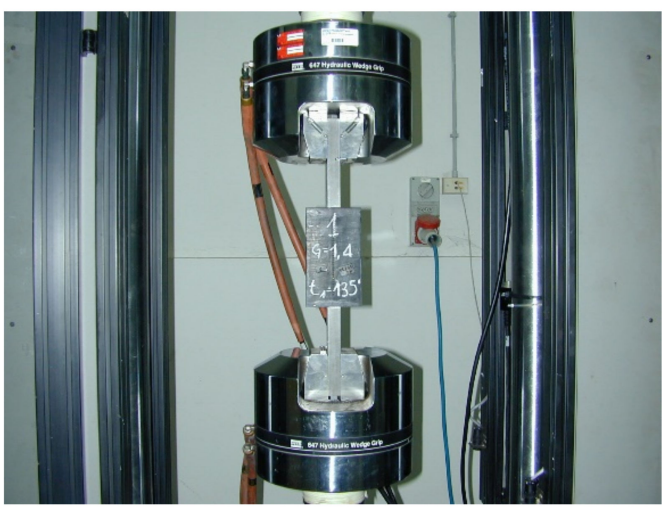

Figure 3. Test setup.

\subsection{Experimental Results}

Under the application of increasing displacements, the sample first stretches uniformly, then it undergoes necking at an indeterminate region (as in Figure 4a) and finally, the failure occurs in the region of the necking (as in Figure 5a).

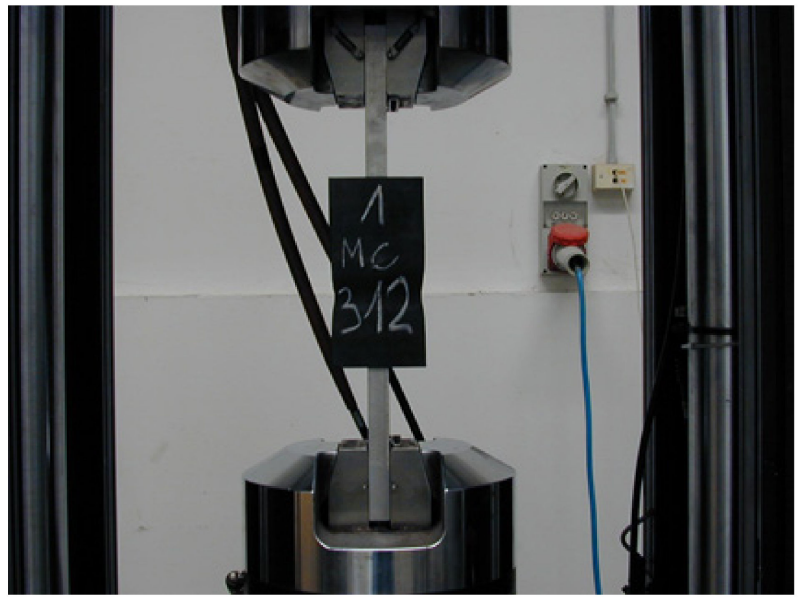

(a)

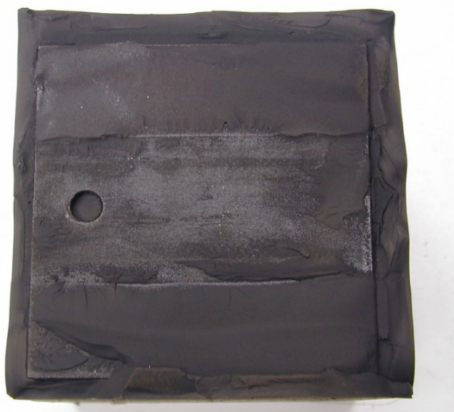

(b)

Figure 4. Sample 11 (a) just before failure; (b) after cohesive failure.

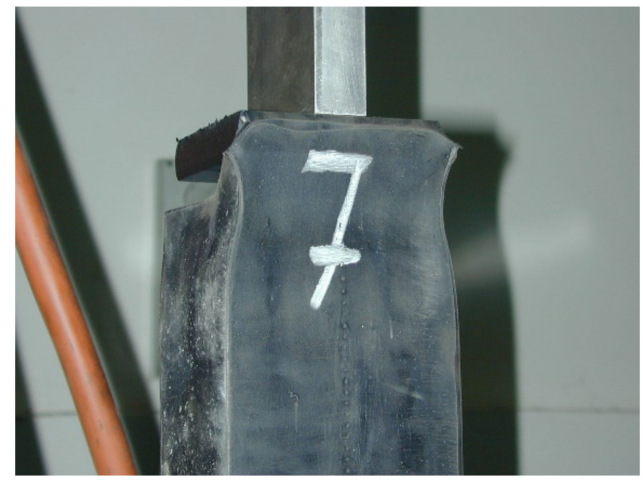

(a)

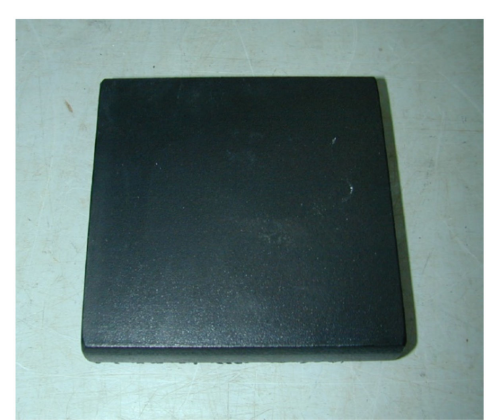

(b)

Figure 5. Sample 7 (a) at failure; (b) after adhesive failure.

The experimental force-displacement diagrams of all samples were recorded and are reported below in the discussion of the experimental results. The following significant parameters were extrapolated from these diagrams: 
- $F_{\max }$, which is the maximum force that the sample withstands

- $\quad d_{F_{\max }}$, which is the elongation of the sample for $F_{\max }$

- $\quad d_{u}$, which is the elongation of the sample at failure, calculated as the elongation corresponding to a force reduced of $5 \%$ with respect to $F_{\max }$.

The values of these parameters for all of the tested samples are shown in Tables 2-7 together with the type of failure exhibited by the sample. The sample failures are classified as follows [6]:

- Cohesive failure is when the failure occurs only inside the elastomer due to the tensile stresses. This failure produces a ruptured surface within the elastomer with tear damage, as it can be seen in Figure $4 \mathrm{~b}$. In samples that undergo this type of failure, the deformation and strength properties of the elastomer are completely exploited before the occurrence of adhesive failure.

- Adhesive failure occurs when the failure involves the bonding surface between the steel and elastomer and results in a detachment, without any damage to the elastomer component. It results in the elastomeric layer detaching from the steel layer (Figure 5b) and occurs when the bond strength between the two layers is not sufficient to attain cohesive failure.

- Mixed failure is manifest by intermediate behaviors (Figure $6 \mathrm{~b}$ ) between the cohesive and adhesive failures described above. In this case, some portions of the sample show rubber detached from steel, and other portions show tear damage.

Table 2. Experimental results for samples 1 to 9.

\begin{tabular}{ccccccc}
\hline Sample & G $[$ MPa] & $\begin{array}{c}\text { Vulcanization } \\
\text { Time [min] }\end{array}$ & $\boldsymbol{F}_{\text {max }}[\mathbf{k N}]$ & $\boldsymbol{d}_{\boldsymbol{F}_{\text {max }}}[\mathbf{m m}]$ & $\boldsymbol{d}_{\boldsymbol{u}}[\mathbf{m m}]$ & Type of Failure \\
\hline 0 & 1.4 & 135 & 36.78 & 1.91 & 4.22 & - \\
$1(\mathrm{cs})$ & 1.4 & 135 & 37.18 & 1.60 & 7.82 & Cohesive \\
2 & 1.4 & 150 & 35.86 & 0.90 & 3.71 & Mixed \\
3 & 1.4 & 180 & 37.65 & 1.10 & 2.11 & Adhesive \\
$4(\mathrm{cs})$ & 0.8 & 135 & 24.77 & 3.31 & 5.12 & Cohesive \\
5 & 0.8 & 150 & 14.62 & 1.30 & 3.01 & Adhesive \\
6 & 0.8 & 180 & 12.60 & 26.30 & - & Mixed \\
$7(\mathrm{cs})$ & 0.4 & 135 & 18.07 & 10.32 & 12.43 & Adhesive \\
8 & 0.4 & 150 & 17.57 & 13.42 & 17.93 & Mixed \\
9 & 0.4 & 180 & 14.12 & 8.43 & 15.03 & Not detected \\
\hline
\end{tabular}

(cs) = control sample.

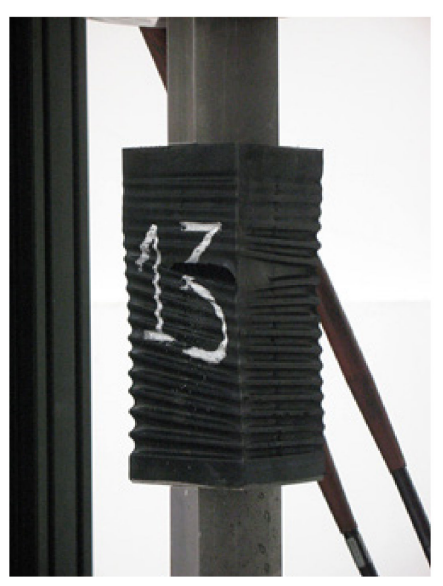

(a)

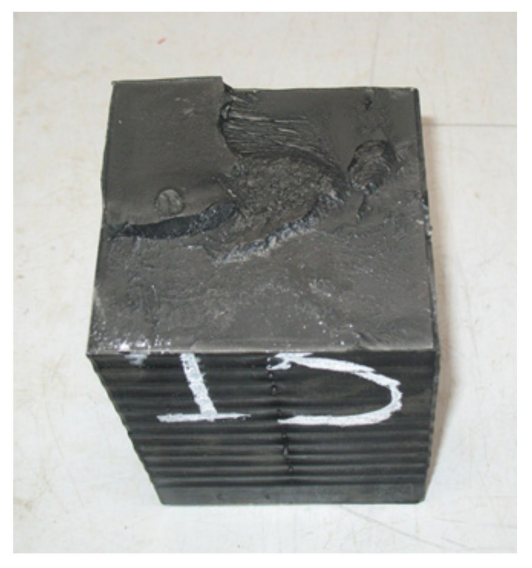

(b)

Figure 6. Sample 18b (a) at failure; (b) after mixed failure. 
Table 3. Experimental results for samples 1 and 10.

\begin{tabular}{cccccc}
\hline Sample & Sandblasting Material & $\boldsymbol{F}_{\max }[\mathrm{kN}]$ & $\boldsymbol{d}_{\boldsymbol{F}_{\max }}[\mathrm{mm}]$ & $\boldsymbol{d}_{\boldsymbol{u}}[\mathrm{mm}]$ & Type of Failure \\
\hline $1(\mathrm{cs})$ & Grit & 37.18 & 1.60 & 7.82 & Cohesive \\
10 & Corundum & 36.32 & 1.00 & 3.21 & Mixed \\
\hline
\end{tabular}

Table 4. Experimental results for samples 1, 14a and 14b.

\begin{tabular}{cccccc}
\hline Sample & Covering Layer & $\boldsymbol{F}_{\boldsymbol{m a x}}[\mathbf{k N}]$ & $\boldsymbol{d}_{\boldsymbol{F}_{\max }}[\mathbf{m m}]$ & $\boldsymbol{d}_{\boldsymbol{u}}[\mathbf{m m}]$ & Type of Failure \\
\hline $1(\mathrm{cs})$ & Yes & 37.18 & 1.60 & 7.82 & Cohesive \\
$14 \mathrm{a}$ & No & 35.34 & 1.41 & 2.91 & Mixed \\
$14 \mathrm{~b}$ & No & 33.42 & 1.71 & 3.12 & Mixed \\
& Average $14 a$ and $14 b$ & 34.38 & 1.56 & 3.02 & \\
\hline
\end{tabular}

Table 5. Experimental results for samples 14a, 14b, 15a and 15b.

\begin{tabular}{cccccc}
\hline Sample & Oily Component & $\boldsymbol{F}_{\max }[\mathbf{k N}]$ & $\boldsymbol{d}_{\boldsymbol{F}_{\max }}[\mathbf{m m}]$ & $\boldsymbol{d}_{\boldsymbol{u}}[\mathbf{m m}]$ & Type of Failure \\
\hline 14a $(\mathrm{cs})$ & Yes & 35.34 & 1.41 & 2.91 & Mixed \\
$14 \mathrm{~b}(\mathrm{cs})$ & Yes & 33.42 & 1.71 & 3.12 & Mixed \\
& Average $14 a$ and $14 b$ & 34.38 & 1.56 & 3.02 & \\
$15 \mathrm{a}$ & No & 37.22 & 1.20 & 30.45 & Cohesive \\
$15 \mathrm{~b}$ & No & 35.23 & 1.28 & 17.80 & Cohesive \\
& Average 15a and $15 b$ & 36.23 & 1.24 & 24.13 & \\
\hline
\end{tabular}

Table 6. Experimental results for samples 14a, 14b, 16a, 16b, 17a, 17b, and 17a, 16b.

\begin{tabular}{ccccccc}
\hline Sample & Refreshed Elastomer & Bridge Layer & $\boldsymbol{F}_{\max }[\mathrm{kN}]$ & $\boldsymbol{d}_{\boldsymbol{F}_{\max }}[\mathrm{mm}]$ & $\boldsymbol{d}_{\boldsymbol{u}}[\mathrm{mm}]$ & Type of Failure \\
\hline $14 \mathrm{a}(\mathrm{cs})$ & No & No & 35.34 & 1.41 & 2.91 & Mixed \\
$14 \mathrm{~b}(\mathrm{cs})$ & No & No & 33.42 & 1.71 & 3.12 & Mixed \\
& & Average 14a and $14 b$ & 34.38 & 1.56 & 3.02 & \\
16a & Yes & No & 34.29 & 123.34 & 123.61 & Abnormal \\
$16 \mathrm{~b}$ & Yes & No & 34.16 & 1.51 & 4.52 & Adhesive \\
$17 \mathrm{cs})$ & No & Yes & 34.77 & 3.31 & 2.32 & Adhesive \\
$17 \mathrm{~b}(\mathrm{cs})$ & No & Yes & 32.83 & 2.61 & 4.91 & Adhesive \\
& & Average 17a and 17b & 33.80 & 2.96 & 3.62 & \\
$18 \mathrm{a}$ & Yes & Yes & 29.50 & 2.41 & 16.63 & Mixed \\
$18 \mathrm{~b}$ & Yes & Yes & 32.33 & 1.61 & 36.46 & Cohesive \\
& & Average 18a and 18b & 30.92 & 2.01 & 26.55 & \\
\hline
\end{tabular}

Table 7. Experimental results for samples 4, 11 and 12.

\begin{tabular}{ccccccc}
\hline Sample & Rubber Type & Compound Type & $\boldsymbol{F}_{\max }[\mathbf{k N}]$ & $\boldsymbol{d}_{\boldsymbol{F}_{\max }}[\mathrm{mm}]$ & $\boldsymbol{d}_{\boldsymbol{u}}[\mathrm{mm}]$ & Type of Failure \\
\hline 4 (cs for 12) & natural & - & 24.77 & 3.31 & 5.12 & Cohesive \\
11 & - & non-dissipative & 30.38 & 7.62 & 11.03 & Cohesive \\
12 (cs for 11) & synthetic & dissipative & 29.34 & 23.34 & 29.25 & Mixed \\
\hline
\end{tabular}

\section{Discussion}

Tables 2-7 show that the minimum strength of the tested samples were $14.12 \mathrm{kN}, 12.60 \mathrm{kN}$ and $29.50 \mathrm{kN}$ for the group of samples with $\mathrm{G}=0.4 \mathrm{MPa}, \mathrm{G}=0.8 \mathrm{MPa}$ and $\mathrm{G}=1.4 \mathrm{MPa}$, respectively. Since UNI EN 15129 [15] limits the value of tensile stresses acting on elastomeric isolators to 2G, by multiplying $2 \mathrm{G}$ by the sample base area $\left(0.1 \times 0.1 \mathrm{~m}^{2}\right)$, the isolators should have strengths at least equal to $8 \mathrm{kN}, 16 \mathrm{kN}$ and $28 \mathrm{kN}$, respectively. The experimental results shown in Tables 2-7 are reported in Figures 7-9, together with the relative minimum tensile strength provided by [15]. It should be noted that the minimum strength for samples of the group with $\mathrm{G}=0.8 \mathrm{MPa}(12.60 \mathrm{kN}$, sample 6$)$ is lower than $16 \mathrm{kN}$. Moreover, the strength of another sample in this group, sample 5 (14.62 kN), is also lower than this value. This suggests that these two samples do not satisfy the standards required by UNI EN 15129, as can also be seen in Figure 8. On the contrary, the strengths of all the other samples 
are greater than the required values, as can be seen in Figures 7-9. This indicates that these samples have satisfactory tensile behavior according to UNI EN 15129. Figure 7 shows several samples with a deformation capacity much greater than that of other specimens. These are samples $15 a$ and $15 b, 18 a$ and $18 \mathrm{~b}$, which are identical pairs, and the average of their experimental outcomes is reported in the figure. The particular behavior of samples $15 \mathrm{a}$ and $15 \mathrm{~b}$ is discussed in Section 4.4 , and that of samples $18 \mathrm{a}$ and $18 \mathrm{~b}$ is discussed in Section 4.6.

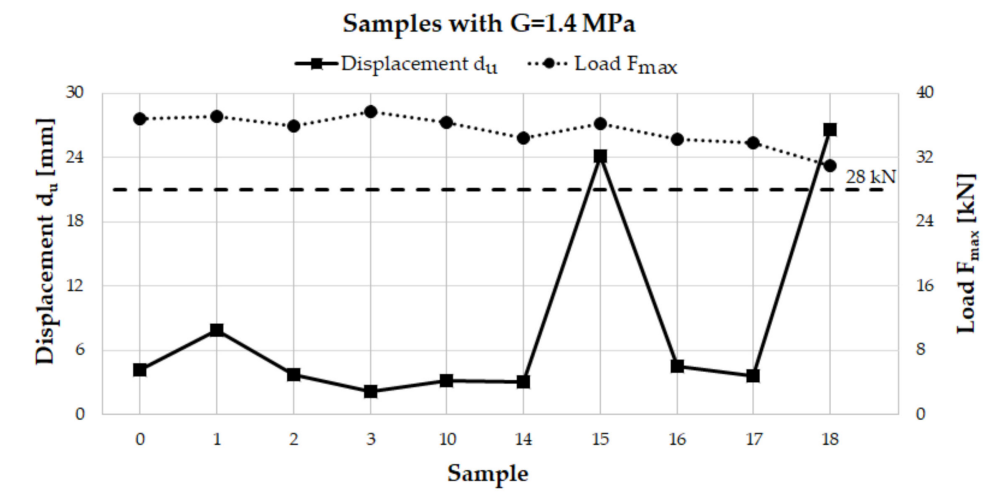

Figure 7. Test results in terms of displacements and loads for samples with $\mathrm{G}=1.4 \mathrm{MPa}$.

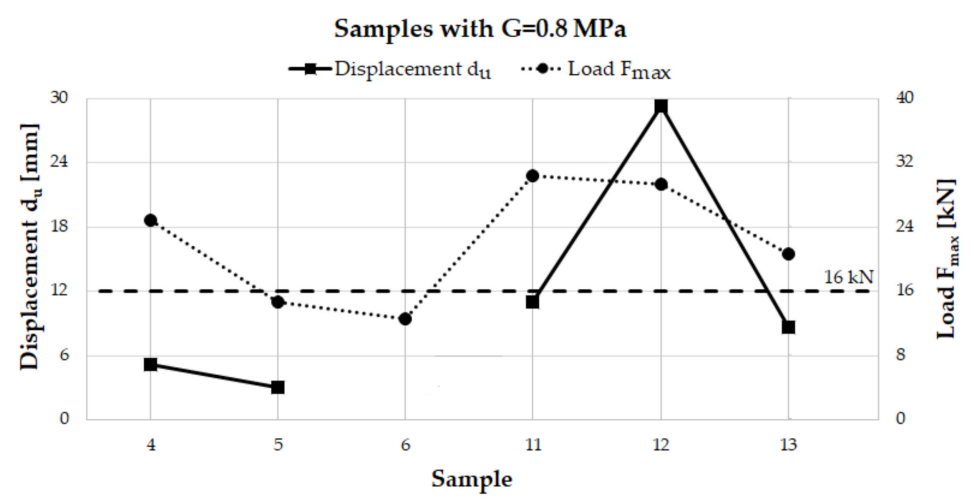

Figure 8. Test results in terms of displacements and loads for samples with $\mathrm{G}=0.8 \mathrm{MPa}$.

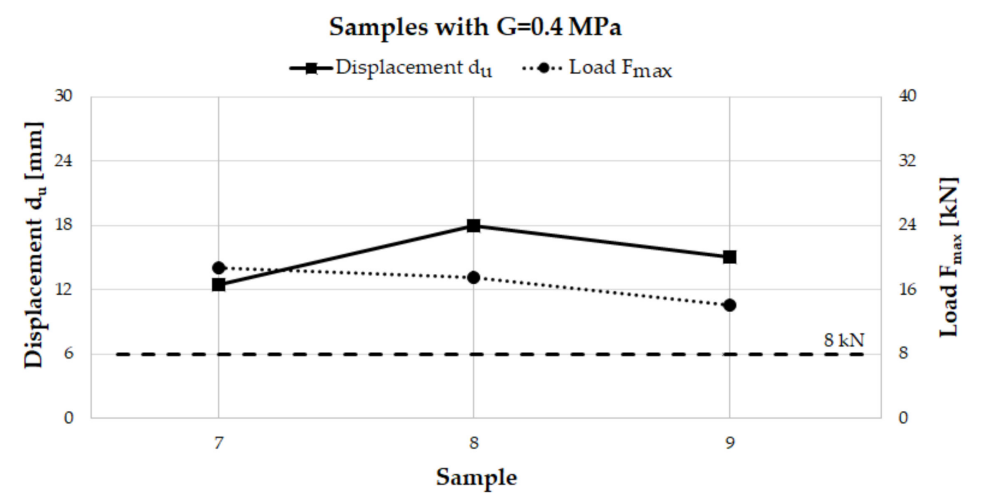

Figure 9. Test results in terms of displacements and loads for samples with $\mathrm{G}=0.4 \mathrm{MPa}$.

As regards Figure 8, the scattered results suggest that further experimentation should be performed to verify the materials' behavior and to exclude the possibility of a problem during the manufacturing of the samples.

In the following, we compare the tensile test results between groups of samples that differ in only one characteristic, in order to detect the influence of such a characteristic on the adhesion between elastomer and steel layers. It will also enable an understanding of the reason for the low strength of samples 5 and 6 . 


\subsection{Influence of Increase in Vulcanization Time}

In Figures 10-12, the tensile load-displacement response curves of samples according to the variation in vulcanization time are reported for direct comparison. The control sample is identified by the initials (cs) placed next to the specimen number.

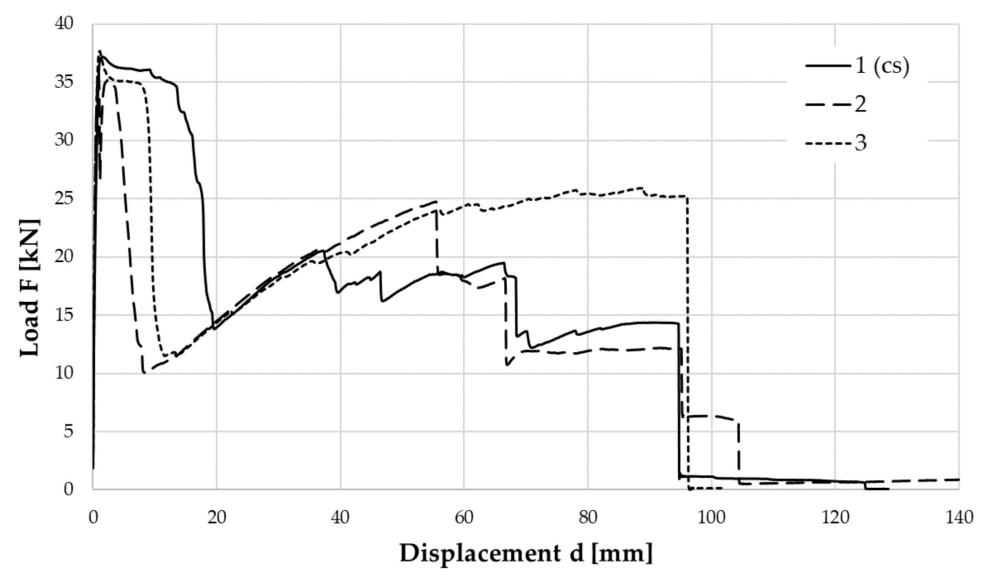

Figure 10. Tensile load-displacement response curves of samples 1, 2, $3(\mathrm{G}=1.4 \mathrm{MPa})$.

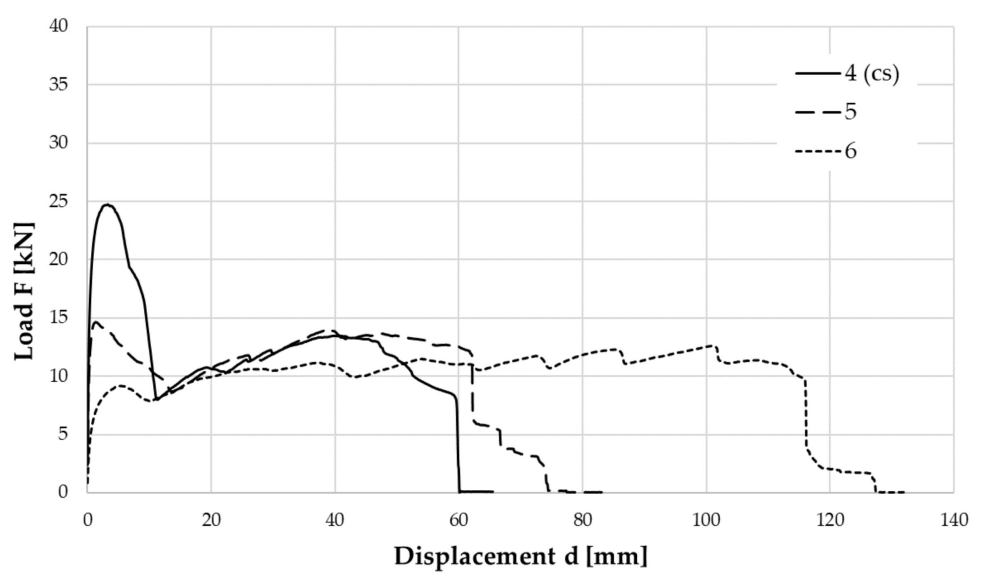

Figure 11. Tensile load-displacement response curves of samples 4, 5, $6(\mathrm{G}=0.8 \mathrm{MPa})$.

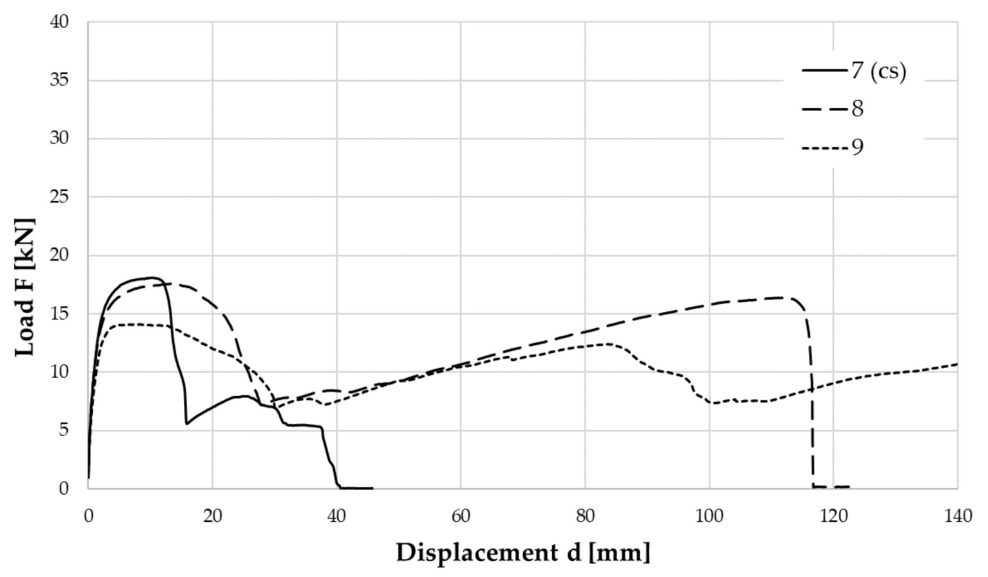

Figure 12. Tensile load-displacement response curves of samples $7,8,9(\mathrm{G}=0.4 \mathrm{MPa})$.

These figures are followed by Table 2, where the features of the compared samples are reported together with the experimental results in terms of the maximum recorded force, $\mathrm{F}_{\max }$, the corresponding displacement, $\mathrm{d}_{\text {Fmax }}$, and the ultimate displacement, $\mathrm{d}_{\mathrm{u}}$. 
In Figures 13-15, the test results in terms of maximum stress and maximum elongation of samples with the same shear modulus $G$ and with different vulcanization times are shown.

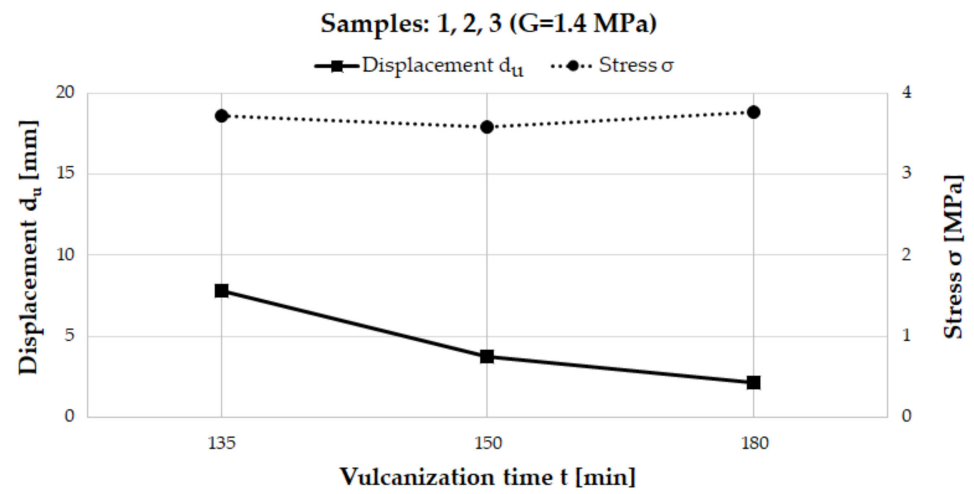

Figure 13. Test results in terms of displacement and stress for samples $1,2,3(\mathrm{G}=1.4 \mathrm{MPa})$ for various vulcanization times.

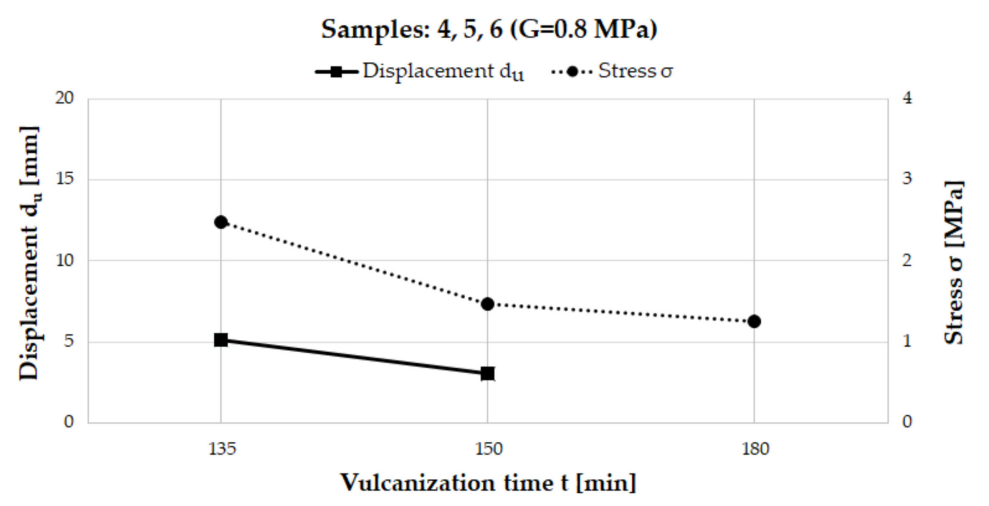

Figure 14. Test results in terms of displacement and stress for samples $4,5,6$ ( $\mathrm{G}=0.8 \mathrm{MPa}$ for various vulcanization times.

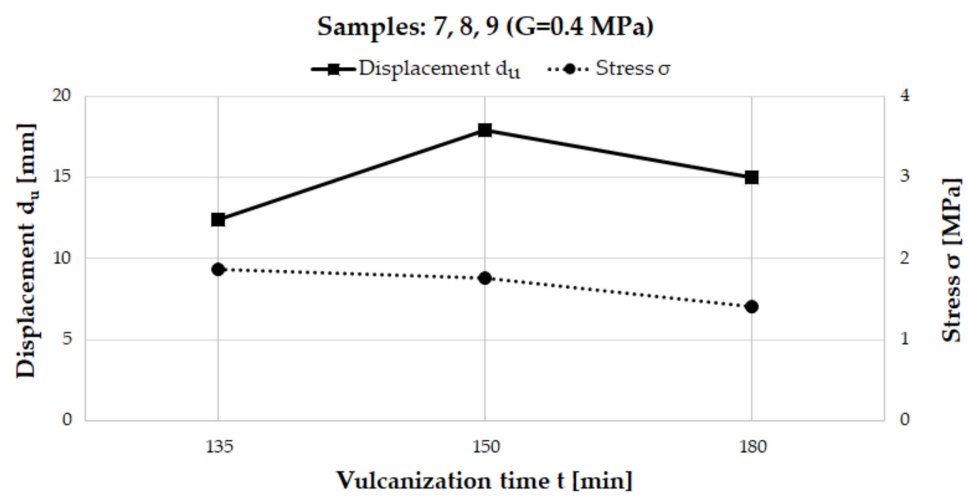

Figure 15. Test results in terms of displacement and stress for samples $7,8,9(\mathrm{G}=0.4 \mathrm{MPa})$ for various vulcanization times.

From Figures 10-15 and Table 2, it can be seen that there was no improvement in the strength of samples with a vulcanization time of 150 and $180 \mathrm{~min}$ compared to the control sample, which had a vulcanization time of $135 \mathrm{~min}$. Indeed, in general the strength stayed almost the same or decreased. Regarding the deformation capacity, only the elongations in samples 6, 8 and 9 were greater than that of the control sample, however, the strength decreased in these samples.

The longer vulcanization time probably influences the degradation of elastomer material due to the longer exposure to high temperature. Moreover, the steel layers, being made of metal, can store the heat within them. Since the layers are packed in elastomer, they release the heat to the elastomer 
over a period of time even longer than the vulcanization time. This may result in degradation of the elastomer. The two described phenomena can explain the observed drop in the maximum load.

The samples that showed cohesive failure are samples 1 and 4 , which also performed the best in terms of maximum strength because the strength of the elastomer prevents premature detachment of the elastomer from the reinforcement layers. The other samples show adhesive or mixed failures.

The conclusion regarding the influence of increasing the vulcanization is that this parameter does not improve the adhesion between elastomer and steel layers. On the contrary, for specimens 5 and 6 , it reduced their capacity in terms of strength, which decreased to values lower than the strength required by UNI EN 15129 [15] under tensile action, as already pointed out (Figure 8).

\subsection{Influence of Sandblasting with Corundum}

The tensile load-displacement response curves of samples when different material was used for sandblasting are reported in Figure 16 for direct comparison.

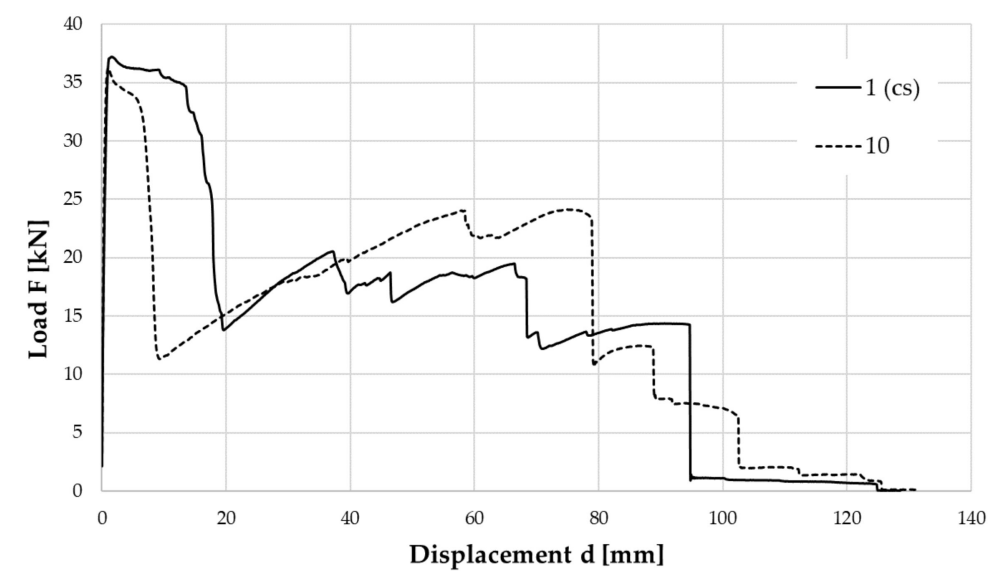

Figure 16. Tensile load-displacement response curves of samples 1 and 10, which were sandblasted with standard grit and corundum, respectively.

This figure is followed by Table 3, where the features of the compared samples are shown together with the experimental results.

The reinforcements of sample 10 were sandblasted with corundum. As it can be seen from the relevant data in Table 3 and the curves in Figure 16, the performance of this sample was not better than that of the control sample (sample 1), but it was quite similar. Therefore, it can be concluded that the use of corundum sandblasting does not improve the adhesion between elastomer and steel layers.

\subsection{Influence of External Covering Layer}

In Figure 17, the tensile load-displacement response curves of samples with or without an external covering layer are reported for direct comparison.

This figure is followed by Table 4, where the features of the compared samples are reported together with the experimental results. For the identical samples, the average values of the parameters were calculated and reported below the two samples.

Samples 14a and 14b, which were realized without an external covering layer, show slightly worse behavior in terms of strength compared to the control specimen (sample 1). However, their behavior is worse in terms of ultimate elongation and also the type of failure. Hence, it can be concluded that the absence of the covering layer does not improve the adhesion between the layers, but, instead, worsens it. 


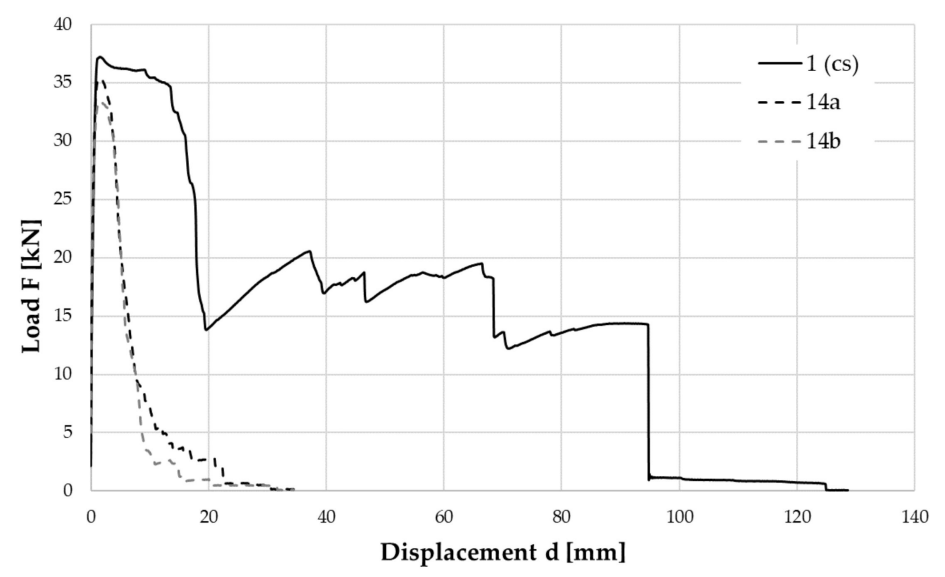

Figure 17. Tensile load-displacement response curves of sample 1 with an external covering layer and samples $14 \mathrm{a}$ and $14 \mathrm{~b}$ without an external covering layer.

\subsection{Influence of Elastomer without an Oily Component}

In Figure 18, the tensile load-displacement response curves of samples that have or do not have the oily component in their elastomer layers are reported for direct comparison.

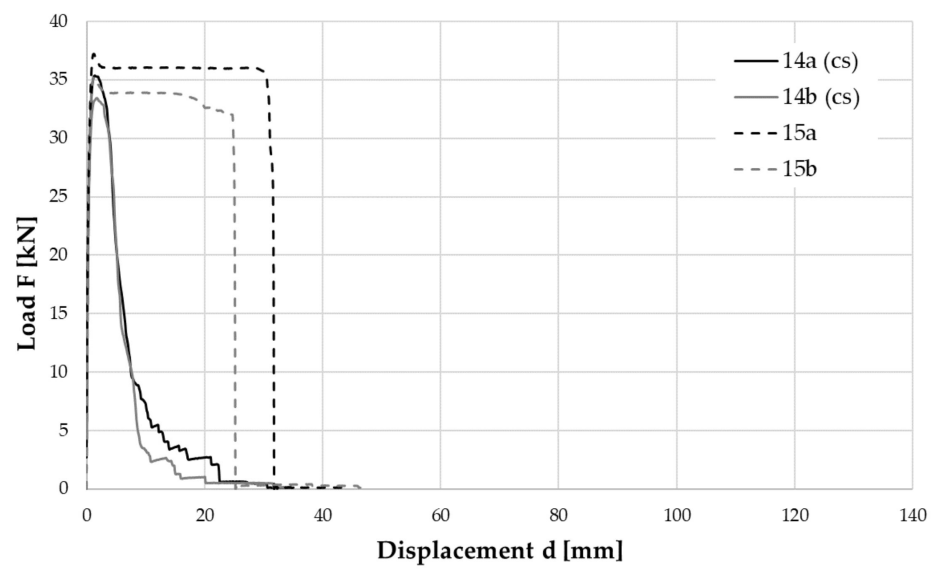

Figure 18. Tensile load-displacement response curves for samples $14 \mathrm{a}$ and $14 \mathrm{~b}$, with an oily component in the elastomer layers, and samples $15 \mathrm{a}$ and $15 \mathrm{~b}$, without it.

This figure is followed by Table 5, where the features of the compared samples are reported together with the experimental results.

Samples $15 \mathrm{a}$ and $15 \mathrm{~b}$ were built using elastomer layers without an oily component. In comparison with samples 14a and 14b (Table 5), which are considered as control samples in this case, samples 15a and $15 \mathrm{~b}$ show a slightly greater strength, and clearly better ultimate elongation (almost 8 times greater) and type of failure. In fact, as can be seen from Figure 18, the force-displacement curves recorded for samples $15 \mathrm{a}$ and $15 \mathrm{~b}$ are more "ductile" in the sense that these samples are able to maintain the maximum strength for a larger range of displacements, in comparison to samples $14 \mathrm{a}$ and $14 \mathrm{~b}$. This happens because the failure in samples $15 \mathrm{a}$ and $15 \mathrm{~b}$ occurs on the elastomer side, which can undergo great elongation before breaking, while samples 14a and 14b show a mixed failure. In light of this, it can be concluded that using elastomer layers without oily component. improves the adhesive behavior of the elastomeric isolators, because it helps to avoid adhesive failure. Moreover, the increase in the isolator ductility increases the dissipative capacity of the isolator, which is very important for reducing the seismic action on a building. 


\subsection{Influence of Use of Refreshed Elastomer}

In Figures 19 and 20, the tensile load-displacement response curves of samples with refreshed elastomer layers are compared to control samples, whose elastomer layers have not been refreshed.

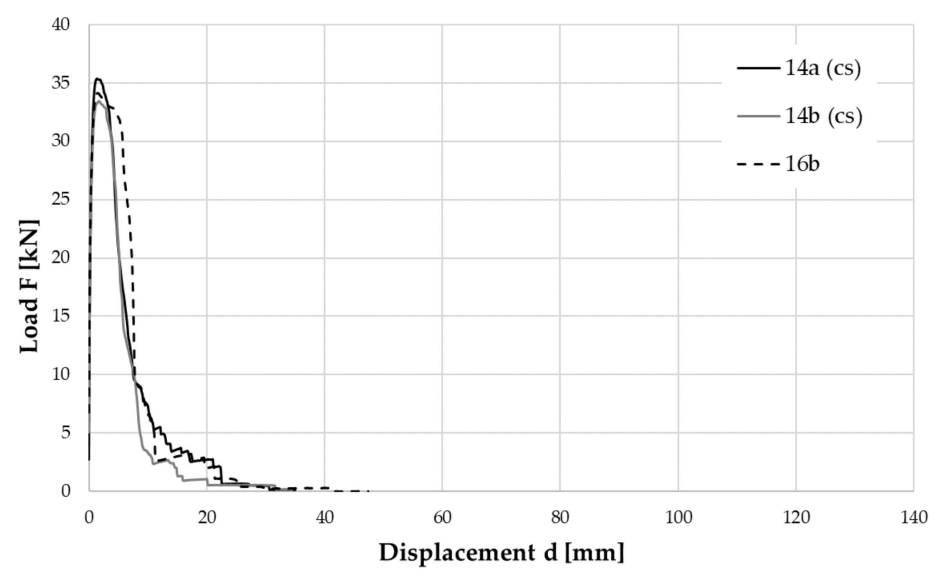

Figure 19. Tensile load-displacement response curves for samples 14a and 14b, without refreshed elastomer layers, and sample 16b, with refreshed elastomer layers.

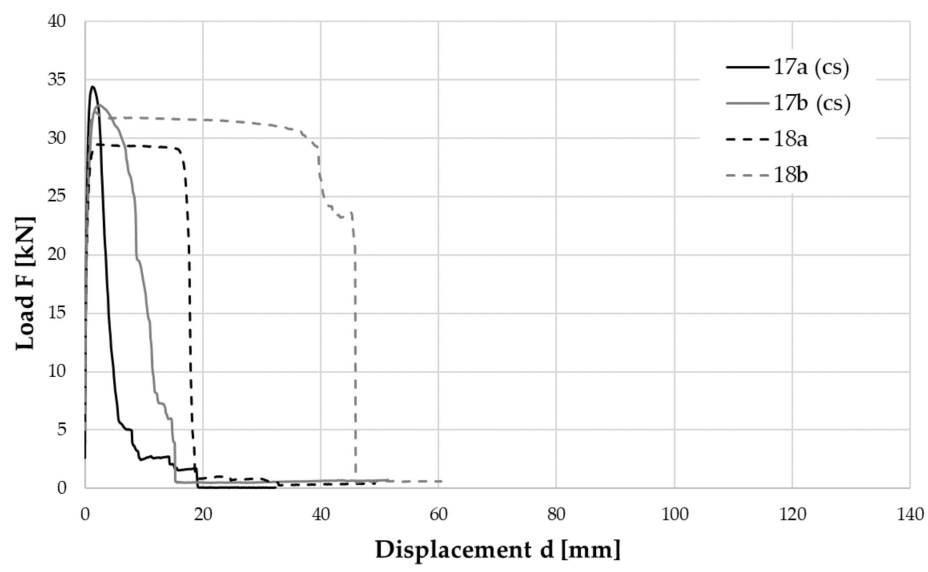

Figure 20. Tensile load-displacement response curves for samples $17 \mathrm{a}$ and $17 \mathrm{~b}$, without refreshed elastomer layers, and 18a and 18b, with refreshed elastomer layers.

These figures are followed by Table 6, where the features of the compared samples are reported together with the experimental results.

Samples 16a and 16b and 18a and 18b were built using elastomer layers that were refreshed before being put into the mold for vulcanization to eliminate the problem of oily component on the surfaces. Sample 16a was excluded from the study, because its behavior was anomalous.

Sample 16b, in comparison with samples $14 a$ and $14 b$, which were the control samples in this case, shows almost the same strength, and only a little more ultimate elongation, as it can be seen in Figure 19.

Samples 18a and 18b, in comparison with samples 17a and 17b, which were used as the control samples, show a little less strength on average, but greater elongation capacity (Figure 20).

Hence, since the observations were ambiguous, the only certainty is that the use of refreshed elastomer does not negatively influence the adherence between steel and elastomer and it may improve the elongation capacity, i.e., the ductility, and consequently, the dissipative capacity of the isolator. 


\subsection{Influence of Bridge Layer}

In Figures 21 and 22, the tensile load-displacement response curves of samples with bridge layers are compared to the control samples, which do not have bridge layers.

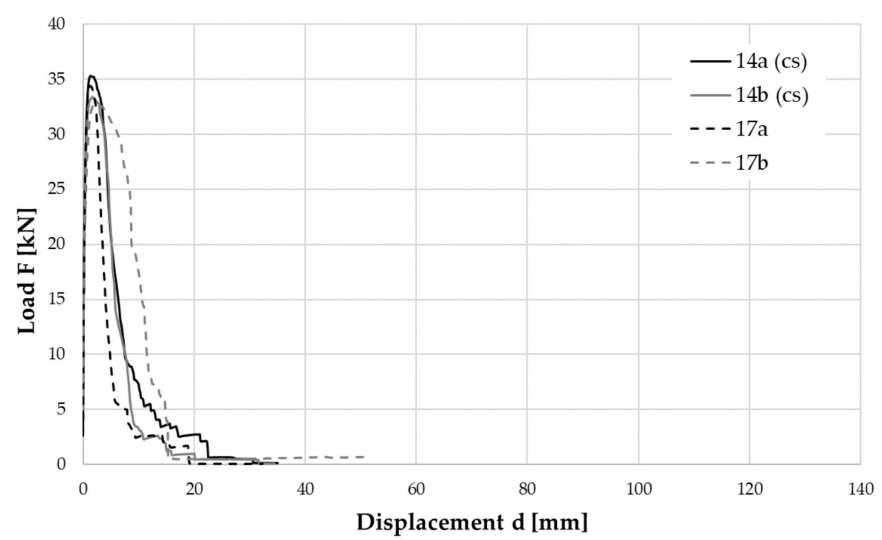

Figure 21. Tensile load-displacement response curves for samples $14 \mathrm{a}$ and $14 \mathrm{~b}$, without bridge layers and $18 \mathrm{a}$ and $18 \mathrm{~b}$, with bridge layers.

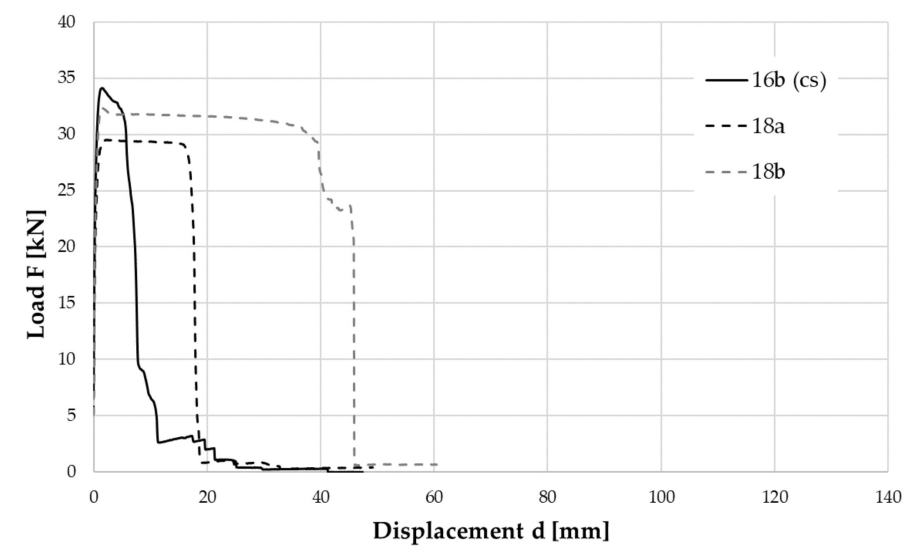

Figure 22. Tensile load-displacement response curves of sample 16b, without bridge layers, and samples $17 \mathrm{a}$ and $17 \mathrm{~b}$, with bridge layers.

The features of the compared samples and the relative experimental results can be found in Table 6.

Samples $17 \mathrm{a}$ and $17 \mathrm{~b}$ and $18 \mathrm{a}$ and $18 \mathrm{~b}$ were built using a "bridge layer", which was put between the mastic and elastomer layer. Compared to the control samples (samples 14a and 14b), samples $17 \mathrm{a}$ and $17 \mathrm{~b}$ show almost the same behavior (Figure 21), both in terms of strength and elongation capacity. Samples 18a and 18b show a little less strength, but greater elongation capacity (Figure 22) in comparison with their control samples (samples 16a and 16b).

Hence, since the observations are ambiguous, the only certainty is that the use of bridge layers does not negatively influence the adherence between steel and elastomer, and it may improve the elongation capacity, i.e., the ductility, and consequently, the dissipative capacity of the isolator.

\subsection{Influence of Base Rubber Type and Non-Dissipative Behavior of the Elastomer Compound}

In Figures 23 and 24, the tensile load-displacement response curves of samples with elastomer layers made of different types of compound are reported for direct comparison. 


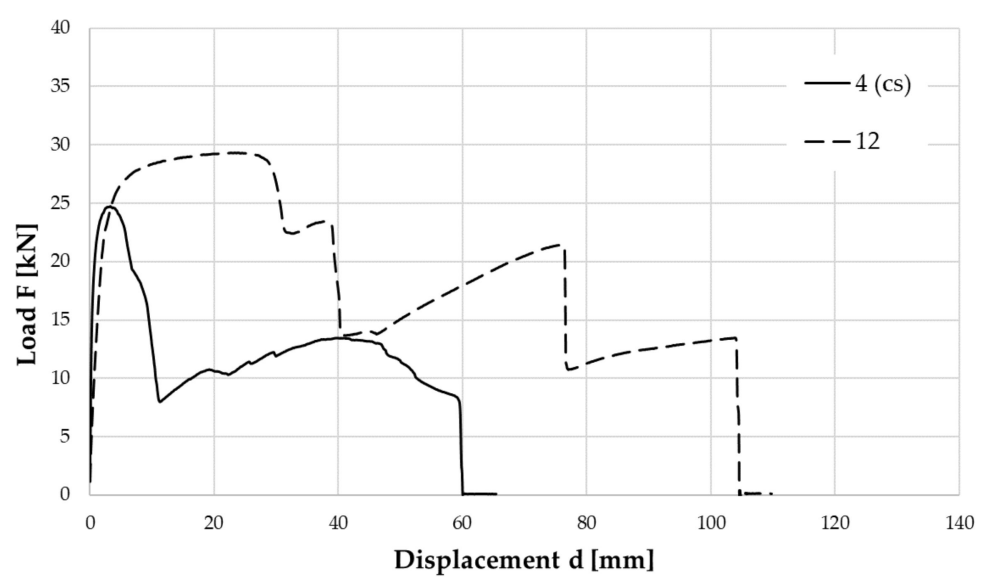

Figure 23. Tensile load-displacement response curves of samples 4 and 12, with elastomer layers made by natural and synthetic rubber-based compounds, respectively.

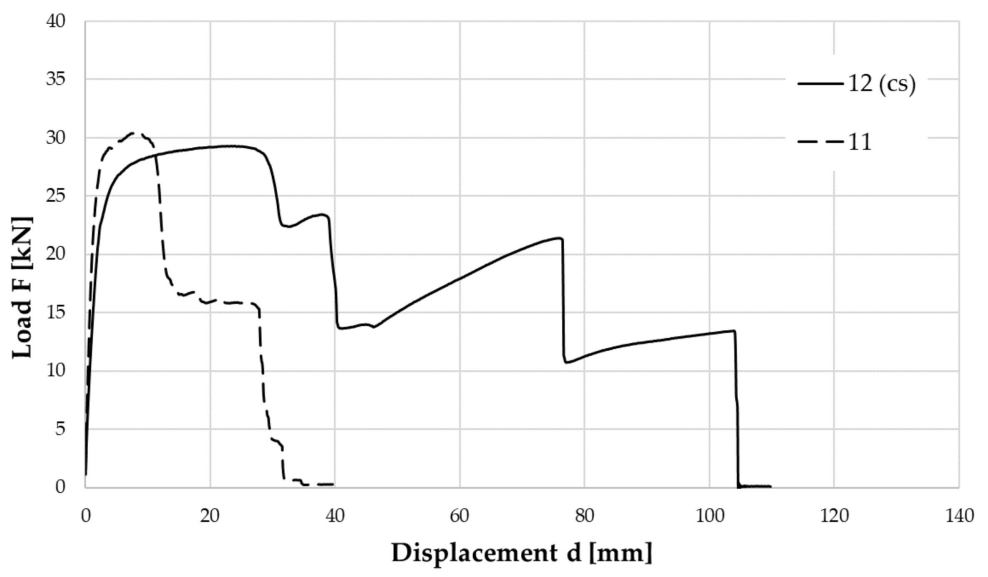

Figure 24. Tensile load-displacement response curves of samples 11 and 12, with elastomer layers made by non-dissipative and dissipative compounds, respectively.

These figures are followed by Table 7, where the features of the compared samples are reported together with the experimental results.

With regard to the influence of the rubber used to produce the elastomer, sample 12, which was built using a synthetic rubber-based compound was compared to sample 4, which was built using a natural rubber-based compound. As it can be seen in Table 7 and the curves in Figure 23, sample 12 shows greater strength and much greater ultimate elongation compared to sample 4 . This happens even if sample 12 undergoes mixed failure, while sample 4 shows cohesive failure.

It can be concluded that synthetic rubber provides the isolator with greater ductility, i.e., dissipative capacity in comparison with natural rubber.

With regard to the influence of the non-dissipative behavior of the elastomer compound, sample 11 , which was built using a non-dissipative compound was compared to sample 12, which is the control specimen in this case. As it can be seen from Table 7 and the curves in Figure 24, sample 11 has slightly greater strength, but much less ultimate elongation in comparison to sample 12 . Hence, as was expected, the non-dissipative compound gives the isolator lower ductility.

\section{Conclusions}

The adhesion behavior between elastomer and steel layers of elastomeric isolators was investigated through tensile tests performed on 24 square-based samples. The effects of varying some of the manufacturing processes on the performance of the tested samples was analyzed. On the basis of the obtained experimental results, the authors make the following conclusions: 
- All samples, apart from samples 5 and 6, show satisfactory tensile behavior according to UNI EN 15129 , i.e., they have a strength that is greater than $2 \mathrm{G}$.

- An increase in vulcanization time does not improve the adhesion between elastomer and steel layers, on the contrary, for samples 5 and 6, it reduced their capacity in terms of strength.

- The use of corundum in the sandblasting process does not improve or worsen the adhesion between elastomer and steel layers.

- The absence of an external covering layer does not improve the adhesion between the layers, but instead, it worsens it in terms of the deformation capacity and failure type.

- Using elastomer layers without an oily component. improves the adhesive behavior of elastomeric isolators, because they are able to avoid adhesive failure. Moreover, it increases the isolator ductility, i.e., its dissipative capacity.

- The use of refreshed elastomer and the use of bridge layers does not negatively influence the adhesion between steel and elastomer, whereas they may improve the elongation capacity, i.e., the ductility, and consequently, the dissipative capacity of the isolator.

- Synthetic rubber provides the isolator with greater ductility, i.e., dissipative capacity, in comparison with natural rubber, and greater strength.

- A non-dissipative compound gives the isolator lower ductility without affecting its strength.

Author Contributions: Conceptualization, M.P.; methodology, M.P.; software, F.P.; investigation, G.F. and M.P.; resources, G.R.; data curation F.P.; writing—original draft preparation, F.P., G.F. and M.P.; writing-review and editing, M.P.; visualization, F.P.; supervision, G.R.; project administration, G.R. All authors have read and agreed to the published version of the manuscript.

Funding: This research received no external funding.

Acknowledgments: ILPEA Industries S.p.A is gratefully acknowledged for providing the samples. The authors also acknowledge engineer Guido Tognan for collaborating in the arrangement of the test setup.

Conflicts of Interest: The authors declare no conflict of interest.

\section{References}

1. Kelly, J.M. Earthquake-Resistant Design with Rubber, 2nd ed.; Springer: London, UK, 1997.

2. Naeim, F.; Kelly, J.M. Design of Seismic Isolated Structures from Theory to Practice; John Wiley \& Sons: New York, NY, USA, 1999; 304p.

3. Pauletta, M. Method to design fiber-reinforced elastomeric isolators (U-FREIs) and application. Eng. Struct. 2019, 197, 109366. [CrossRef]

4. Pauletta, M.; Cortesia, A.; Pitacco, I.; Russo, G. A new bi-linear constitutive shear relationship for Unbonded Fiber-Reinforced Elastomeric Isolators (U-FREIs). Comp. Struct. 2017, 168, 725-738. [CrossRef]

5. Russo, G.; Pauletta, M.; Cortesia, A. A Study on Experimental Shear Behavior of Fiber-Reinforced Elastomeric Isolators with Various Fiber Layouts, Elastomers and Aging Conditions. Eng. Struct. 2013, 52, $422-433$. [CrossRef]

6. Pauletta, M.; Battocchio, E.; Russo, G. A weathering steel elastomer joint for the connection between new and existing bridges. Eng. Struct. 2015, 105, 264-276. [CrossRef]

7. Russo, G.; Pauletta, M. Sliding instability of fiber-reinforced elastomeric isolators in unbonded applications. Eng. Struct. 2013, 48, 70-80. [CrossRef]

8. Russo, G.; Pauletta, M.; Cortesia, A.; Del Bianco, A. Experimental behavior of carbon fiber reinforced isolators. In Proceedings of the 2008 Seismic Engineering International Conference Commemorating the 1908 Messina and Reggio Calabria Earthquake, Reggio Calabria, Italy, 8-11 July 2008.

9. Pauletta, M.; Cortesia, A.; Russo, G. Roll-out instability of small size fiber-reinforced elastomeric isolators in unbonded applications. Eng. Struct. 2015, 102, 358-368. [CrossRef]

10. Angeli, P.; Russo, G.; Paschini, A. Carbon fiber-reinforced rectangular isolators with compressible elastomer: Analytical solution for compression and bending. Int. J. Solids Struct. 2013, 50, 3519-3527. [CrossRef]

11. Maureira, N.; de la Llera, J.; Oyarzo, C.; Miranda, S. A nonlinear model for multilayered rubber isolators based on a co-rotational formulation. Eng. Struct. 2017, 131, 1-13. [CrossRef] 
12. Keda, Y.; Nagahashi, S. Earthquake response characteristics on uplift of multilayered elastomeric isolator of base isolated high-rise building with setback elevation: A study on the effects of simultaneous action input from horizontal and vertical ground motion on earth quake response characteristics of base isolated high-rise building, Part 2. J. Struct. Constr. Eng. 2010, 75, 913-922.

13. Mitoulis, S.A. Uplift of elastomeric bearings in isolated bridges subjected to longitudinal seismic excitations. Struct. Infrastruct. Eng. 2015, 11, 1600-1615. [CrossRef]

14. Tubaldi, E.; Mitoulis, S.A.; Ahmadi, H.; Muhr, A. A parametric study on the axial behaviour of elastomeric isolators in multi-span bridges subjected to horizontal seismic excitations. Bull. Earthq. Eng. 2016, 14, 1285-1310. [CrossRef]

15. European Committee for Standardization. UNI EN 15129-2009. Anti-Seismic Devices; European Committee for Standardization: Brussels, Belgium, 2009.

16. Kalfas, K.N.; Mitoulis, S.A.; Katakalos, K. Numerical study on the response of steel-laminated elastomeric bearings subjected to variable axial loads and development of local tensile stresses. Eng. Struct. 2017, 134, 346-357. [CrossRef]

Publisher's Note: MDPI stays neutral with regard to jurisdictional claims in published maps and institutional affiliations.

(C) 2020 by the authors. Licensee MDPI, Basel, Switzerland. This article is an open access article distributed under the terms and conditions of the Creative Commons Attribution (CC BY) license (http://creativecommons.org/licenses/by/4.0/). 